\title{
Culture numérique et conception architecturale... Retour vers le futur
}

Le Séminaire de Conception Architecturale Numérique (SCAN), devenu un des rendezvous de la communauté francophone de l'architecture, rassemble des chercheurs, des enseignants et des praticiens autour de questionnements portant sur les implications du numérique en conception architecturale. Après avoir abordé les thèmes de l'esquisse numérique (2005), de l'image numérique (2007), des approches environnementales (2009), de l'espace collaboratif (2010), des complexités des modèles de l'architecture numérique (2012), de(s) l'interaction(s) des maquettes numériques (2014), de la mesure et démesure du projet (2016), de la dynamique immersion-émersion (2018), l'édition 2020 de SCAN propose de faire un arrêt sur image en se plongeant dans les débuts du numérique en conception architecturale, d'observer son processus d'évolution, et d'approcher les enjeux paradoxaux qui mènent la conception architecturale numérique de ce 21e siècle.

Dès les années 70, les prémisses de l'introduction de l'informatique en architecture pointaient déjà dans les laboratoires de recherche des écoles d'architecture formant ainsi les premières communautés francophones qui poseront les bases d'un questionnement sur la conception architecturale numérique bien avant la mutation de la profession. Que ce soit sur des questions analytiques et computationnelles, sur des questions patrimoniales, sur des questions théoriques et formelles, sur des questions d'enseignement et de conception, voire sur des questions philosophiques, la fin des années 1980 et le début des années 1990 voient progressivement émerger le numérique en architecture. A ce moment, toute une série de projets et de publications surgissent sur la scène médiatique architecturale et participent à poser, un à un, les jalons d'un nouveau territoire. Pour mémoire et à titre d'exemples, en 1990, William Mitchell écrit "The Logic of Architecture"; en 1991 les Liquid architectures de Marcos Novak dématérialisent l'expérience spatiale en projetant l'observateur dans des mondes 3D numériques autonomes; en 1991 toujours, Christian Père modélise en 3D l'Abbaye de Cluny; en 1993, Greg Lynn est éditeur du numéro d'Architectural Design "Folding in Architecture"; en 1994, Bernard Tschumi lance le Paperless Studio à l'université de Columbia. Les bases d'un domaine de recherche, d'expérimentation et de pratiques nouvelles s'établissent; une génération d'architectes déploie, alors avec un enthousiasme résolu, une approche inédite de l'architecture qui va amener à redéfinir la conception architecturale de façon profonde dans les décennies qui suivront, jusqu'à aujourd'hui.

Riche de productions foisonnantes et de bouleversements épistémologiques essentiels, le numérique en architecture, hétérogène et polymorphe, est maintenant trentenaire. Il est 
mature et a désormais la possibilité de se retourner vers son histoire. Comme cela a déjà été fait par Greg Lynn avec l'exposition « Archéologie du numérique » au Centre Canadien d'Architecture en 2013, dorénavant, c'est au tour de « SCAN’20 - Culture numérique et conception architecturale ... Retour vers le futur » de regarder dans le rétroviseur afin de mieux appréhender la conception, et les perspectives qui s'offrent à la recherche, dans, et pour le futur. Quelles étaient les promesses du passé ? Quelle est la réalité du présent ? Et sur quel avenir est-il raisonnable de spéculer ? L’hyper connectivité a transformé l'assistance à la modélisation - la CAO par exemple - en assistance numérique dans la consultation participative pour la conception - par exemple la maquette numérique. De l'esquisse à la ville numérique en passant par l'architecture, le développement fulgurant des réseaux de communication a bouleversé nos modes de pensées plongeant les concepteurs dans une complexité qui voit l'émergence de nouvelles manières de penser le cycle de vie du bâtiment sous l'angle de vue du numérique - les SmartCities, les BIMs, la fabrication 3D, etc. Par ailleurs, les modèles 3D et les images numériques, générées à profusion par la technologie contemporaine, ont redéfini les contours des modes d'existence et de perception de l'architecture. Que sont devenus les enjeux culturels et sociaux d'une architecture dont le rapport à la matérialité s'avère de plus en plus ambigu et questionnant ? Que devient la conception architecturale à l'heure du Big Data, de la généralisation des TIC et du développement du BIM et des FabLab ? Le numérique des débuts, spécifique et avant-gardiste, a-t-il évolué vers des pratiques génériques, vers un numérique totalement englobant - émergeant à présent sous le terme post-numérique - dont on cherche aujourd'hui à tracer les contours et à définir les spécificités ?

Cette édition de SCAN est composée de vingt-quatre articles qui s'attaquent chacun à l'une ou plusieurs de ces questions. Quatre domaines principaux sont abordés.

Tout d'abord, les contributions qui adressent directement la question du Retour vers le futur, d'un point de vue épistémologique, ouvrent le séminaire. Ces réflexions cherchent à situer de façon explicite certains enjeux du numérique contemporain en les mettant en relation avec des précédents historiques. Les trois premiers articles s’intéressent à l'évolution des outils et des pratiques. Charlotte Dautremont, Vincent Martin et Sylvie Jancart observent deux décennies d'évolution du travail collaboratif et multidisciplinaire permis par les outils numériques au sein d'une agence. Laila Koubaa, Khaoula Raboudi et Abdelkader Ben Saci relatent également un travail rétrospectif, mais cette fois sur les approches numériques du modèle de l'enveloppe solaire et de son évolution. Thierry Ciblac, quant à lui, spécule sur les potentialités qui pourraient émerger de la transposition dans le numérique d’un domaine datant du XIXe siècle : la statique graphique. Les trois articles suivants abordent des questions d'histoire et de théorie. Julien Rippinger sonde les fondements de l'infographie qui ont été posés par les outils Sketchpad et Sketchpad III dans les années 1960, et s'interroge sur la possibilité d'un modèle alternatif de l'infographie qui s'appuierait sur des principes de géométrie descriptive. Marie-Pascale Corcuff propose de comparer la démarche de recherche et de conception de l'architecte allemand Frei Otto au mode de pensée qui régit la conception d’algorithmes numériques, et entreprend de comparer ces deux démarches sur base de trois cas d'études. Enfin, Sébastien Bourbonnais revient sur un évènement ponctuel mais néanmoins important de l'introduction du numérique dans le processus conceptuel : l'épisode du Fractal Studio, en 1988, sous la 
houlette de Chris I. Yessios et de Peter Eisenman, évènement qui participera à établir les enjeux formels spécifiques à l'architecture dite numérique des décennies qui suivront.

Ensuite vient l'incontournable BIM, ou Building Information Modeling, domaine de pointe issu des questions de conception assistée et de collaboration augmentée qui ont animé la réflexion du numérique en architecture dès sa genèse, et dont l'importance s’accroît à chaque édition de SCAN. Le BIM a désormais sa propre histoire et peut émerger en tant que terrain épistémologique spécifique. Trois articles abordent d'ailleurs le BIM sous l'angle de l'épistémologie de la conception architecturale numérique. S'intéressant précisément à l'importance croissante du BIM, non en conception architecturale mais bien dans le domaine de l'étude patrimoniale, Alexandra Stoleru et Kévin Jacquot se basent sur la bibliométrique et la scientométrie pour mesurer l'impact et identifier les tendances du HBIM, ou Historic Building Information Modeling, dans la production scientifique récente. Après cela, Aurélien Catros s’intéresse au statut et à la nature du modèle du BIM, en situant ce dernier par rapport au concept plus général de modèle scientifique. Il s’appuie également sur diverses comparaisons historiques pour identifier l'évolution de ce concept de modèle, en évoquant notamment les rôles descriptifs et exploratoires des maquettes de la Renaissance. Toujours dans une approche rétrospective, Léa Sattler, Samir Lamouri et Robert Pellerin étudient l'intégration des activités de conception architecturale en un système numérique centralisé pendant la période qui précède l'avènement du BIM, avant les années 2000. Ils procèdent pour cela d'une analyse de la littérature scientifique relative au sujet. Les trois articles suivants examinent la façon dont le BIM s'est implanté, dont les architectes et les agences d'architecture se l'approprient dans leur production courante, et concernent donc l'observation des pratiques. Tout d'abord, Elodie Hochscheid et Gilles Halin étudient, par le biais d'une enquête et d'un questionnaire, le degré l'adoption du BIM par les agences sur le territoire français, ainsi que le niveau d'utilisation qu'ont adopté ces dernières, le cas échéant. Elodie André, Hafida Boulekbache et Mohamed-Anis Gallas s'interrogent sur la façon dont le BIM pourrait articuler et stimuler d'autres aspects très importants de la conception architecturale contemporaine et de la construction, à savoir ceux relatifs au développement durable. Pour terminer, Roland Billon, Isabelle Fasse et Thierry Parinaud s'intéressent aux aspects collaboratifs du modèle numérique et questionnent l'interopérabilité effective mise en place par le standard IFC (Industry Foundation Classes). Ils observent les difficultés que ce mode de travail engendre entre les différents intervenants du projet et proposent une piste logicielle pour les résoudre.

Un autre aspect tout aussi fondamental de la conception numérique en architecture, la question du processus, est ensuite abordée. Les trois premières contributions étudient les mécanismes de cognition et d'interaction qui prennent place entre les concepteurs et leur outil. Tout d'abord, Elizabeth Mortamais s'intéresse au nouveau statut que le numérique confère au point de départ de tout projet : l'esquisse. Un regard rétrospectif et critique sur les outils numériques qui ont caractérisé jadis ce stade initial de toute production, ainsi que l'observation des pratiques et des outils plus récents, lui permet de proposer une réflexion sur la nature contemporaine de cette étape du processus créatif. Gaëlle Baudoux, Xaviéra Calixte et Pierre Leclercq travaillent également sur la phase de l'esquisse, et sur la façon dont se forment les idées pendant cette phase du processus. Par l'observation des pratiques au sein d'un atelier de Master, ils identifient des procédures génériques (ou patterns) dans 
la façon de combiner différents outils de conception, numériques ou non, dans une perspective de conception collaborative. Enfin, Anabelle Rahhal, Charline Berthelot, Stéphane Safin et Pierre Leclercq étudient l'autre extrémité du processus de la production architecturale, à savoir la phase de construction. Ils envisagent divers modes d'annotation, notamment par le biais d'une tablette graphique, pour permettre à l'architecte d'enrichir la maquette numérique sur base des observations faites sur le chantier. Les trois contributions suivantes transposent la réflexion dans la phase opérationnelle et appliquée du processus : la modélisation. Quentin Meurisse, Isabelle De Smet, Hadrien Mélot, David Laplume, Thomas Brihaye, Cédric Rivière, Emeline Coszach, Jérémy Cenci, Sesil Koutra et Vincent Bécue utilisent la modélisation procédurale pour proposer un prototype de programme permettant la génération automatique de tissu urbain avec, comme objectif, la densification urbaine durable. Adrien Coppens, Tom Mens et Mohamed-Anis Gallas transposent la phase de modélisation dans un contexte technologique très avancé, combinant la modélisation paramétrique et la réalité virtuelle. Plongeant le concepteur au sein d'un dispositif immersif en 3D, ils observent les outils et les possibilités de manipulation directe des formes et de l'espace en temps réel par l'utilisateur. Alexandre Van Dongen, Sébastien Lambot, Peter Eeckhout et David Lo Buglio utilisent le relevé GPR (radar pénétrant le sol) et le relevé photogrammétrique aérien pour la numérisation et l'étude dans un contexte archéologique de grande échelle. Sur base de données massives, ils réfléchissent à une méthodologie de modélisation combinant données géométriques et études historiques pour formuler des hypothèses sur la conception urbaine qui a régi le site de Pachacamac au Pérou.

Les questions relatives à l'enseignement et à la méthodologie de la conception constituent le dernier - mais néanmoins fondamental - domaine de réflexion de cette édition de SCAN. Comme nous l'avons constaté dans une série de contributions précédentes, la notion de processus est inhérente à l'utilisation des outils numériques en conception architecturale. Ceci met donc en exergue des questions de méthodologie spécifiques au domaine, ainsi que la question de l'enseignement de telles méthodologies. Les trois contributions suivantes se focalisent d'ailleurs sur des dispositifs d'enseignement spécifiques à cette matière. Ainsi, Nawri Khamallah, Renaud Pleitinx et Luca Sgambi connectent la technologie de pointe à un domaine de connaissance datant de la fin du XVIIIe siècle ; à l'aide d'un dispositif immersif de réalité virtuelle, ils proposent une nouvelle approche pour la compréhension et l'enseignement des systèmes projectifs abstraits et parfois complexes de la géométrie descriptive. Toujours à propos de dispositifs immersifs, Sandro Varano, Nicolas Descamps et Eric Touvenot relatent leur expérience de mise en place d'un EVE (Educational Virtual Environment) pour confronter les étudiants à diverses situations rencontrées lors du processus de conception architecturale. Enfin, Christine Wacta utilise la théorie des jeux associée à l'utilisation de téléphones portables pour développer une méthode d'enseignement ludique et intuitive susceptible d'intéresser les générations actuelles d'étudiants. Après la mise au point de dispositifs techniques spécifiques, les trois derniers articles relatent des retours d'expérience et de la théorie, toujours relative à l'enseignement du numérique en architecture. Tout d'abord, Laurent Lescop et Olivier Chamel proposent de comparer la façon dont les différents outils numériques sont mobilisés par les étudiants dans le contexte d'une école française et d'une école américaine. Ensuite, Mélina Ramondenc et Philippe Marin explorent, avec leurs 
étudiants, l'architecture modulaire de Pascal Häusermann, Claude Costy et Jean-Louis Chanéac: à travers un processus de reconstruction 3D et de modélisation procédurale paramétrique, ils génèrent de nouvelles configurations architecturale par la recombinaison des éléments dessinés par les trois architectes dans les années 1960 et 1970 . Pour conclure, Philippe Marin revient sur l'histoire des technologies de l'information qui ont permis la genèse des outils utilisés aujourd'hui par les architectes, et sur la façon dont ces outils ont forgé la culture numérique contemporaine.

Le comité de programme tient à remercier chaleureusement tous les auteurs qui ont proposé une contribution à cette édition de SCAN, ainsi que tous les membres du comité scientifique qui ont, par leurs doubles relectures à l'aveugle, permis d'opérer la sélection proposée dans le présent ouvrage. Les conditions de travail difficiles liées à la crise sanitaire de la Covid-19 ont certainement imposé des efforts supplémentaires, tant aux auteurs qu'aux relecteurs. Néanmoins, ces investissements combinés ont permis de mener à bien le processus éditorial. S'en est suivie une édition de SCAN inédite car intégralement tenue dans un environnement virtuel, via visioconférence.

Denis Derycke, Geneviève Martin, Samia Ben Rajeb, Sylvie Jancart, Pierre Leclercq. 